Stefan Hamm • Bénédicte Dehouck • Jörg Kraus •

Karen Wolburg-Buchholz • Hartwig Wolburg •

Werner Risau · Roméo Cecchelli • Britta Engelhardt •

Marie-Pierre Dehouck

\title{
Astrocyte mediated modulation of blood-brain barrier permeability does not correlate with a loss of tight junction proteins from the cellular contacts
}

Received: 16 May 2003 / Accepted: 10 October 2003 / Published online: 13 November 2003

(C) Springer-Verlag 2003

\begin{abstract}
In the central nervous system (CNS) complex endothelial tight junctions (TJs) form a restrictive paracellular diffusion barrier, the blood-brain barrier (BBB). Pathogenic changes within the CNS are frequently accompanied by the loss of BBB properties, resulting in brain edema. In order to investigate whether $\mathrm{BBB}$ leakiness can be monitored by a loss of TJ proteins from cellular borders, we used an in vitro BBB model where brain endothelial cells in co-culture with astrocytes form a tight permeability barrier for ${ }^{3} \mathrm{H}$-inulin and ${ }^{14} \mathrm{C}$-sucrose. Removal of astrocytes from the co-culture resulted in an increased permeability to small tracers across the brain endothelial cell monolayer and an opening of the TJs to horseradish peroxidase as detected by electron microsco-
\end{abstract}

This work is dedicated to the memory of Werner Risau (died 13.12.1998), who initiated this collaboration

S. Hamm · B. Dehouck · J. Kraus · K. Wolburg-Buchholz · B. Engelhardt

Max Planck Institute for Physiological and Clinical Research, Bad Nauheim, Germany

J. Kraus · B. Engelhardt

Max Planck Institute for Vascular Biology,

Münster, Germany

B. Dehouck · R. Cecchelli · M.-P. Dehouck

Unité mixte Institut Pasteur de Lille-Université d'Artois, U.A. 2465-Faculté des Sciences Jean Perrin,

Lens, France

B. Engelhardt $(\square)$

Theodor Kocher Institute, University of Bern,

Freiestrasse 1, 3012 Bern, Switzerland

e-mail: bengel@tki.unibe.ch

Fax: +41-31-6313799

K. Wolburg-Buchholz $\cdot$ H. Wolburg

Institute for Pathology, Tübingen, Germany

Present address:

S. Hamm, Wyeth Vaccine Research,

Pearl River, NY, USA py. Strikingly, opening of the endothelial TJs was not accompanied by any visible change in the molecular composition of endothelial TJs as junctional localization of the TJ-associated proteins claudin-3, claudin-5, occludin, ZO-1 or ZO-2 or the adherens junction-associated proteins $\beta$-catenin or p120cas did not change. Thus, opening of BBB TJs is not readily accompanied by the complete loss of the junctional localization of TJ proteins.

Keywords Claudins - Occludin - Tight junction - Edema formation · Blood-brain barrier · Cell culture (bovine, rat)

\section{Introduction}

Homeostasis of the central nervous system (CNS) microenvironment is essential for its normal function and is maintained by the blood-brain barrier (BBB), which protects the CNS from compositional fluctuations within the blood stream. The BBB is formed by highly specialized endothelial cells, which inhibit the transcellular passage of hydrophilic molecules across the BBB by an extremely low pinocytotic activity and restrict the paracellular diffusion of hydrophilic molecules by an elaborate network of complex tight junctions (TJ) between the endothelial cells (reviewed by Wolburg and Lippoldt 2002). The morphology of BBB TJs resembles those of epithelial TJs, which in ultrathin section electron micrographs appear as a chain of fusion points of the outer plasma membrane leaflet of adjacent cells (Farqhuar and Palade 1963). Despite the structural similarities between BBB and epithelial TJs (Kniesel and Wolburg 2000), BBB endothelial TJs are unique in their sensitivity to ambient factors. Cultured epithelial cells establish a high transepithelial electrical resistance and low permeability in concert with a stable TJ morphology, mimicking the situation in vivo (Gumbiner and Simons 1986). In contrast, cultured BBB endothelium loses many BBB characteristics including the formation of proper TJs and 
therefore of a highly restrictive paracellular permeability barrier. During pathological conditions of the CNS such as inflammation or tumor formation, loss of barrier properties of the highly specialized cerebral endothelium leads to edema formation and exacerbation of clinical disease. Taken together this indicates that integrity of BBB TJs strictly depends on signals provided by the CNS microenvironment (Wolburg et al. 1994).

Neither the cell types within the CNS nor the signals responsible for the maintenance of $\mathrm{BBB}$ characteristics in cerebral capillary endothelium have been characterized to date. Astrocytic endfeet are in close proximity to the endothelial cell plasma membrane and are separated only by the basal lamina. As the astroglial perivascular sheet is a unique feature of CNS capillaries and forms at around the same time as the permeability barrier develops (Phelps 1972), astrocytes and their precursors have been implicated in the induction and the maintenance of the BBB (Goldstein 1988). Several in vitro BBB models, which utilize the co-culture of cerebral brain endothelial cells with primary astrocytes, thus mimicking the in vivo BBB microenvironment, have confirmed this notion (Meresse et al. 1989; Rubin et al. 1991; Wolburg et al. 1994). In most of those models at least certain BBB characteristics such as a permeability barrier, high electrical resistance or even transcytosis of LDL or transferrin (Cecchelli et al. 1999) across the cerebral endothelial monolayer can be demonstrated.

The aim of the present study was to investigate the molecular changes possibly involved in the development of BBB leakiness with special focus on the molecular composition of tight junctions. Protein components associated with endothelial TJs identified to date include cytoplasmic peripheral membrane proteins of the MAGUK family such as ZO-1 and ZO-2 (reviewed by Tsukita et al. 1999). Integral membrane proteins exclusively localized at TJs are occludin (Furuse et al. 1993; Ando-Akatsuka et al. 1996) and the claudins, which comprise a novel gene family of TJ-associated proteins with no sequence homology to occludin (Furuse et al. 1998; Morita et al. 1999a). Mice carrying a null mutation in the occludin gene develop morphologically normal TJs in most tissues including the brain (Saitou et al. 2000), proving that occludin is not essential for proper TJ formation. In contrast, transfection of claudins into fibroblasts induced TJs in the absence of occludin, demonstrating that claudins are essential for TJ induction (Furuse et al. 1999; Morita et al. 1999b). To date, at least 20 members of the claudin family with different tissue distribution have been described (Mitic et al. 2000). In BBB endothelium expression of claudin-1 and the endothelial cell specific claudin-5 has been described (Morita et al. 1999b; Liebner et al. 2000a). In addition, we recently demonstrated the localization of claudin-3 in TJs of cerebral vessels in healthy brain tissue from man and mouse (Wolburg et al. 2003).

To investigate the molecular composition of endothelial TJs in correlation to barrier characteristics of the $\mathrm{BBB}$, we applied an in vitro BBB model where induction and maintenance of a permeability barrier across cerebral endothelial cells was dependent on the presence of astrocytes, thus closely mimicking the situation in vivo. Removal of astrocytes resulted in an increased permeability to small tracers across the monolayer of brain endothelial cells and an opening of the TJs for horseradish peroxidase as detected by electron microscopy. We demonstrate here that surprisingly modulation of the paracellular permeability across the blood-brain barrier in vitro does not readily correlate with the loss of TJ proteins from the endothelial TJs.

\section{Materials and methods}

Antibodies and staining reagents

The following specific primary antibodies were used: rabbit polyclonal anti-human occludin, rabbit polyclonal anti-human $\mathrm{ZO}-1$, rabbit polyclonal anti-canine $\mathrm{ZO}-2$, and rabbit polyclonal anti-mouse claudin-3 and rabbit polyclonal anti-human claudin-1 (Zymed: lot no. 10464713, catalog no. 51-9000) (all Zymed Laboratories, Inc., San Francisco, CA). A rabbit polyclonal antihuman claudin-1 antibody previously sold by Zymed (lot no. 01162834, catalog no. 71-7800, data not shown) was found to be cross-reactive with claudin- 3 . The specificity of the anti-claudin antibodies employed in this study was therefore verified by immunofluorescence staining and Western blot analysis of transfected mouse L cells expressing either murine claudin-1 or murine claudin-3 (data not shown; the transfectants were kindly provided by M. Furuse, Kyoto, Japan). Rabbit polyclonal anti-human von Willebrand factor was obtained from Dako A/S (Glostrup, Denmark); mouse monoclonal anti-mouse $\beta$-catenin (Clone 14) and mouse monoclonal anti-mouse $\mathrm{p} 120^{\mathrm{cas}}$ were obtained from Transduction Laboratories (Lexington, KY). The rabbit polyclonal antiserum raised against mouse claudin-5/TMVCF was kindly provided by D.H. Kalbacher, Tübingen, Germany (Liebner et al. 2000a, 2000b). Secondary antibodies were Cy3-conjugated goat anti-mouse or goat anti-rabbit IgG (Jackson Immunoresearch Laboratories, Inc., West Grove, PA) and Alexa-conjugated goat anti-rabbit IgG (MoBiTec, Göttingen, Germany).

FITC-labeled phalloidin (Sigma, Deisenhofen, Germany) was used to stain the actin cytoskeleton.

Isolation of bovine brain capillary endothelial (BBCE) cells

Bovine brain capillary endothelial cells were isolated as described by Meresse et al. (1989). Using this culture technique with cloned endothelial cells allowed us to obtain highly pure endothelial cell cultures without contaminating pericytes. Passaged BBCE cells were cultured on gelatine coated Petri dishes in Dulbecco's modified Eagle's medium (DMEM; glucose concentration: $1 \mathrm{~g} / \mathrm{l}$ ) supplemented with $10 \%(\mathrm{v} / \mathrm{v})$ heat inactivated newborn calf serum (CS) and $10 \%(\mathrm{v} / \mathrm{v})$ heat inactivated horse serum (HS) (Life Technologies, Karlsruhe, Germany), $2 \mathrm{mM}$ L-glutamine, $50 \mu \mathrm{g} / \mathrm{ml}$ gentamycin and $1 \mathrm{ng} / \mathrm{ml}$ basic fibroblast growth factor (bFGF, Sigma, Deisenhofen, Germany). BBCE cells were used up to passage 7.

\section{Rat astrocyte cultures}

Primary cultures of glial cells were prepared from the cerebral cortex of newborn rats according to the protocol of Booher and Sensenbrenner (1972), where after removal of the meninges, the brain tissue is forced gently through a nylon sieve and plated onto six-well plates (Greiner, Frickenhausen, Germany) at a concentration of $1.2 \times 10^{5}$ cells/ml in DMEM (glucose concentration: $1 \mathrm{~g} / \mathrm{l}$ ) 
Fig. 1 Experimental setup of the in vitro BBB. BBCE cells and rat astrocytes were co-cultured for 10-12 days prior to the assays. Co-culture was either continued or alternatively discontinued by placing the filter inserts in new six-well tissue culture plates without astrocytes. Permeabilities across the BBCE cell monolayers were compared at the timepoints indicated in "Results"

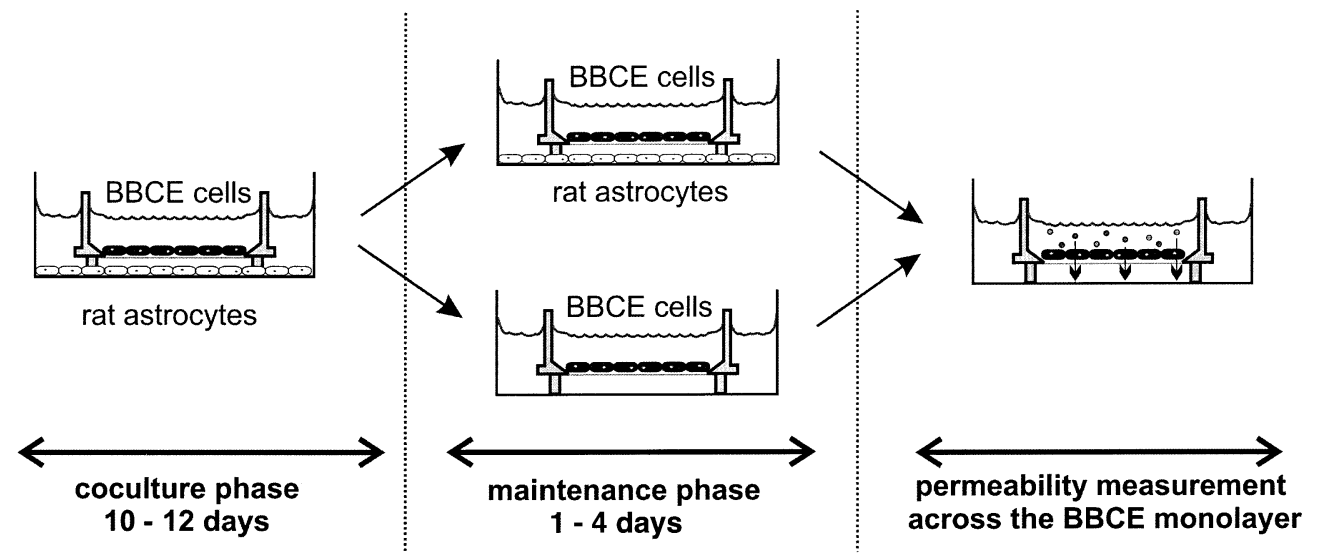

supplemented with $10 \%(\mathrm{v} / \mathrm{v})$ heat inactivated fetal calf serum (PAN, Nürnberg, Germany), $2 \mathrm{mM}$ L-glutamine and $50 \mu \mathrm{g} / \mathrm{ml}$ gentamycin. Three weeks after plating the purity of the astrocyte cultures was confirmed to be $>95 \%$ by immunofluorescence staining for glial fibrillary acidic protein (GFAP) and these cultures were then used in co-culture with bovine brain endothelial cells.

In vitro blood-brain barrier (BBB) culture model

BBCE cells were cultured on collagen-coated filter inserts (Millicell $\mathrm{CM}, 0.4 \mu \mathrm{m}$ pore size, $30 \mathrm{~mm}$ diameter, Millipore, Eschborn, Germany). The filter inserts were coated on the upper side with $150 \mu \mathrm{l}$ rat tail collagen solution (Descamps et al. 1997) supplemented with $10 \times$ DMEM and $0.3 \mathrm{M} \mathrm{NaOH}$. After incubation for $1 \mathrm{~h}$ at $37^{\circ} \mathrm{C}$ the collagen coat was rinsed with $\mathrm{H}_{2} \mathrm{O}$ and PBS. Collagencoated filter inserts were placed into six-well plates containing astrocyte cultures. BBCE cells $\left(4 \times 10^{5}\right.$ cells $\left./ \mathrm{ml}\right)$ were plated on the upper side of the filter insert. The co-culture medium was DMEM (glucose concentration: $1 \mathrm{~g} / \mathrm{l}$ ) supplemented with $10 \%$ (v/v) CS and $10 \%(\mathrm{v} / \mathrm{v}) \mathrm{HS}, 2 \mathrm{mM}$ glutamine, $50 \mu \mathrm{g} / \mathrm{ml}$ gentamycin and $1 \mathrm{ng} / \mathrm{ml}$ bFGF. The co-culture medium was changed every other day. After 3 days BBCE cells reached confluency and were grown for another 7-9 days in co-culture with rat astrocytes to form a tight barrier.

\section{Experimental setup}

After 10-12 days in total, co-culture of BBCE cells with astrocytes was either continued or discontinued by removing the filter inserts from the co-culture and placing them in new six-well plates for the time periods indicated in "Results" (Fig. 1). Paracellular permeabilities of BBCE cell monolayers in the absence or presence of astrocytes using radiolabeled tracers ${ }^{3} \mathrm{H}$-inulin and ${ }^{14} \mathrm{C}$-sucrose were tested in each assay and are not identical for different BBCE clones. Therefore measurements of paracellular permeabilities of BBCE cells using horseradish peroxidase (see below) or immunofluorescence studies (see below) were always accompanied by measurements of paracellular permeability using radiolabeled tracers ${ }^{3} \mathrm{H}$-inulin and ${ }^{14} \mathrm{C}$-sucrose as a reference measurement within each assay. Each assay was repeated with different BBCE clones and performed at least three times.

Transendothelial permeability of BBCE cell monolayers for ${ }^{14} \mathrm{C}$-sucrose and ${ }^{3} \mathrm{H}$-inulin

For quantitative measurement of the paracellular permeability across BBCE cell monolayers, the filter inserts were transferred to six-well plates containing $2.5 \mathrm{ml}$ of HEPES-buffered Ringer's solution per well (lower compartment). At time zero, ${ }^{14} \mathrm{C}$-sucrose $(c=0.05 \mu \mathrm{Ci} / \mathrm{ml})$ and ${ }^{3} \mathrm{H}$-inulin $(c=0.5 \mu \mathrm{Ci} / \mathrm{ml})$ were added in $1.5 \mathrm{ml}$ of HEPES-buffered Ringer's solution on top of the BBCE cell monolayer (upper compartment). Under these conditions hydrostatic pressure did not interfere with the permeability measurement. Plates were incubated at $37^{\circ} \mathrm{C}$ in a $5 \% \mathrm{CO}_{2} / 95 \%$ air atmosphere. Filter inserts were transferred to new six-well plates containing $2.5 \mathrm{ml}$ HEPES-buffered Ringer's solution per well every $10 \mathrm{~min}$ over a time period of $40 \mathrm{~min}$. Values were measured in triplicate. Aliquots were taken from the lower compartment at each timepoint and from the stock solution and radioactivity was determined by using a $\beta$-scintillation counter (Canberra Packard, Frankfurt a.M., Germany). Permeabilities of BBCE cell monolayers for ${ }^{3} \mathrm{H}$-inulin and ${ }^{14} \mathrm{C}$-sucrose were calculated according to the method of Siflinger-Birnboim and colleagues (Siflinger-Birnboim et al. 1987) using the clearance principle as described previously (Cecchelli et al. 1999). All experiments were repeated at least five times.

\section{Ultrastructural analysis of BBCE cell monolayers}

Wheat germ agglutinin conjugated horseradish peroxidase (WGAHRP) (Sigma, Deisenhofen, Germany) was used for ultrastructural analysis of BBCE cell monolayer permeability. Filter inserts with or without $\mathrm{BBCE}$ cells were transferred into six-well plates containing $2.5 \mathrm{ml}$ of HEPES-buffered Ringer's solution (lower compartment), and $1.5 \mathrm{ml}$ of HEPES-buffered Ringer's solution supplemented with $0.1 \mathrm{mg} / \mathrm{ml}$ WGA-HRP was applied to the upper compartment. After $10 \mathrm{~min}$ incubation at $37^{\circ} \mathrm{C}$ in a $5 \% \mathrm{CO}_{2} / 95 \%$ air atmosphere the WGA-HRP solution was removed, and the specimens were washed twice with HEPES-buffered Ringer's solution and fixed for $1 \mathrm{~h}$ at room temperature with $2.5 \%$ glutaraldehyde in $0.1 \mathrm{M}$ sodium cacodylate (pH 7.4). After washing with $0.1 \mathrm{M}$ sodium cacodylate $(\mathrm{pH} 7.4)$, the fixed BBCE cell monolayers were incubated for $30 \mathrm{~min}$ at room temperature with the HRP substrate 3, 3'-diaminobenzidine tetrahydrochloride (1.5 mg/ml; Sigma, Deisenhofen, Germany) and $0.02 \% \mathrm{H}_{2} \mathrm{O}_{2}(\mathrm{v} /$ v) in a TRIS-imidazol buffer $(0.1 \mathrm{M}$ imidazol, $0.05 \mathrm{M}$ TRIS/HCl, $\mathrm{pH}$ 7.0). After washing with $0.1 \mathrm{M}$ sodium cacodylate $(\mathrm{pH} 7.4)$, cells were fixed again for $1 \mathrm{~h}$ at RT with $2.5 \%$ glutaraldehyde in cacodylate buffer. Specimens were washed twice with $0.1 \mathrm{M}$ sodium cacodylate buffer ( $\mathrm{pH} 7.4$ ), postfixed with $1 \% \mathrm{OsO}_{4}$ in $0.1 \mathrm{M}$ cacodylate buffer and dehydrated in an ethanol series. The $70 \%$ ethanol step was saturated with uranyl acetate for contrast enhancement. Dehydration was completed in propylene oxide and samples were embedded in Araldite (Serva, Heidelberg, Germany). Ultrathin sections were mounted on pioloform-coated copper grids and contrasted in part with lead citrate. Specimens were observed and documented with an EM 10A electron microscope (Zeiss, Oberkochen, Germany). Control samples were processed identically except that WGA-HRP was omitted from the solution added to in the upper compartment. No electron-dense HRP reaction product was seen in these specimens. 
Immunofluorescence studies

BBCE cells grown on filter inserts were washed with HEPESbuffered Ringer's solution and fixed with $1 \%$ formaldehyde in PBS at RT or with methanol at $-20^{\circ} \mathrm{C}$. After washing/rehydration with PBS, BBCE cells were permeabilized with $0.1 \%$ Triton X-100 in PBS for $10 \mathrm{~min}$. After washing with PBS the filter membrane with the BBCE cell monolayers was cut out of the filter inserts and transferred into a multiwell plate. Following a 10-min incubation with normal goat serum (NGS), cells were incubated with solutions of the primary antibodies for $1 \mathrm{~h}$ at RT in a humidified chamber; the antibodies were diluted in PBS supplemented with $10 \%$ normal goat serum (NGS). After four PBS washes, cells were incubated with secondary antibodies for $1 \mathrm{~h}$ at RT in the dark. After washing with PBS the filter membranes with the stained BBCE cells on top were mounted with Mowiol 4-88 solution (Calbiochem Co., La Jolla, CA) and the staining was analyzed using an Axiophot microscope (Zeiss, Oberkochen, Germany).

Quantification of immunofluorescence stainings was performed by evaluating the number of high intensity pixels for at least 15 cells using the software Corel Photo Paint 11.

\section{Statistics}

Statistical evaluation of permeability and pixel measures was performed by an unpaired Student's $t$-test using the Macintosh software Instat, with results considered as follows: $p<0.05=$ significant; $p<0.01=$ very significant and $p<0.005=$ extremely significant.

\section{Results}

Maintenance of a permeability barrier across cerebral endothelial cells in vitro is dependent on the presence of astrocytes

An in vitro BBB model was used where cloned bovine brain capillary endothelial (BBCE) cells are grown on collagen-coated filter inserts and co-cultured with primary rat astrocytes in a two-chamber system (Fig. 1). As shown previously (Dehouck et al. 1990), clones of BBCE cells were able to form a tight paracellular permeability barrier for ${ }^{3} \mathrm{H}$-inulin and ${ }^{14} \mathrm{C}$-sucrose in the presence (i.e., $\mathrm{P}_{\mathrm{e}}($ inulin $)=0.07 \times 10^{-3} \pm 0.00 \times 10^{-3} \mathrm{~cm} / \mathrm{min}$ and $\mathrm{P}_{\mathrm{e}}($ sucrose $)=$ $0.16 \times 10^{-3} \pm 0.01 \times 10^{-3} \mathrm{~cm} / \mathrm{min}$ ) but not in the absence of primary rat astrocytes $\left(\mathrm{P}_{\mathrm{e}}(\right.$ inulin $)=0.19 \times 10^{-3} \pm 0.01 \times$ $10^{-3} \mathrm{~cm} / \mathrm{min} ; p<0.001$ and $\mathrm{P}_{\mathrm{e}}($ sucrose $)=0.54 \times 10^{-3} \pm 0.03 \times$ $\left.10^{-3} \mathrm{~cm} / \mathrm{min} ; p<0.001\right)$.

As only BBCEs grown in the presence of astrocytes were induced to form a tight permeability barrier, we asked whether maintenance of the barrier was also dependent on the presence of astrocytes. Therefore, BBCE cells were allowed to form a permeability barrier during 10 days of co-culture with rat astrocytes, then the filter inserts with BBCE cells were removed from the coculture and cultured in the absence of astrocytes for 1-4 consecutive days (Fig. 1). Paracellular permeability of the radiolabeled tracers ${ }^{14} \mathrm{C}$-sucrose and ${ }^{3} \mathrm{H}$-inulin across the BBCE cell monolayers cultured in the absence of rat astrocytes was measured and compared to the permeability measurements obtained for BBCE cells in continued co-culture with rat astrocytes during the entire time period. As soon as $24 \mathrm{~h}$ after removal of astrocytes from the co-culture depending on the investigated BBCE clone, a two- to threefold increase in the permeability of $\mathrm{BBCE}$ cell monolayers for ${ }^{14} \mathrm{C}$-sucrose and ${ }^{3} \mathrm{H}$-inulin could be measured which did not further increase with time (Fig. 2). Therefore, establishment and maintenance of a permeability barrier by BBCE cells in vitro was directly dependent on the presence of astrocytes and made this in vitro BBB model suitable for the study of molecular changes of endothelial TJs associated with alterations of the endothelial paracellular permeability.

The modulation of the in vitro permeability barrier of $\mathrm{BBCE}$ cells is not correlated with a loss of the cortical actin belt

TJs are intracellularly connected with the actin cytoskeleton and its reorganization can cause paracellular permeability changes of cell layers. Therefore we analyzed the actin cytoskeleton of BBCE cells from continued or discontinued co-cultures with astrocytes by using FITCphalloidin. In order to be able to correlate these findings with the permeability barrier characteristics of BBCE cell monolayers, the paracellular permeability for the radioactive tracers ${ }^{3} \mathrm{H}$-inulin and ${ }^{14} \mathrm{C}$-sucrose was measured within the very same experimental setup. BBCE cells grown in co-culture with astrocytes formed a permeability barrier with $P_{\mathrm{e}}$ (inulin) $=0.24 \times 10^{-3} \pm 0.03 \times 10^{-3} \mathrm{~cm} / \mathrm{min}$ and $\mathrm{P}_{\mathrm{e}}$ (sucrose $)=1.05 \times 10^{-3} \pm 0.13 \times 10^{-3} \mathrm{~cm} / \mathrm{min}$, and analysis of actin cytoskeleton revealed a strong cortical actin belt (Fig. 3). Removal of astrocytes from the co-culture resulted in an increase in BBCE cell monolayer permeability with $\mathrm{P}_{\mathrm{e}}$ (inulin) $=1.00 \times 10^{-3} \pm 0.19 \times 10^{-3} \mathrm{~cm} / \mathrm{min}$ $(p<0.005)$ and $\mathrm{P}_{\mathrm{e}}($ sucrose $)=3.57 \times 10^{-3} \pm 0.69 \times 10^{-3} \mathrm{~cm} / \mathrm{min}$ $(p<0.005)$, but the strong cortical actin belt remained unchanged (Fig. 3). Thus, the modulation of the in vitro permeability barrier formed by cerebral endothelial cells was not correlated with a loss of the cortical actin belt at the cellular borders.

Modulation of the paracellular permeability barrier of $\mathrm{BBCE}$ cells in vitro at the ultrastructural level

We next asked if the increased permeability observed in BBCE cell monolayers after termination of the co-culture with astrocytes was associated with differences in the integrity of BBCE cell-to-cell contacts. Therefore we analyzed at the ultrastructural level the passage of wheat germ agglutinin-horseradish peroxidase conjugates (WGA-HRP) across the BBCE cell monolayer. This ultrastructural analysis allowed us to determine whether the increased permeability across BBCE cell monolayers after termination of the co-culture was solely due to a higher paracellular diffusion rate of the tracers or also mediated by endothelial pinocytosis resulting in an increased transcellular transport of tracers. In order to correlate these observations with permeability barrier 

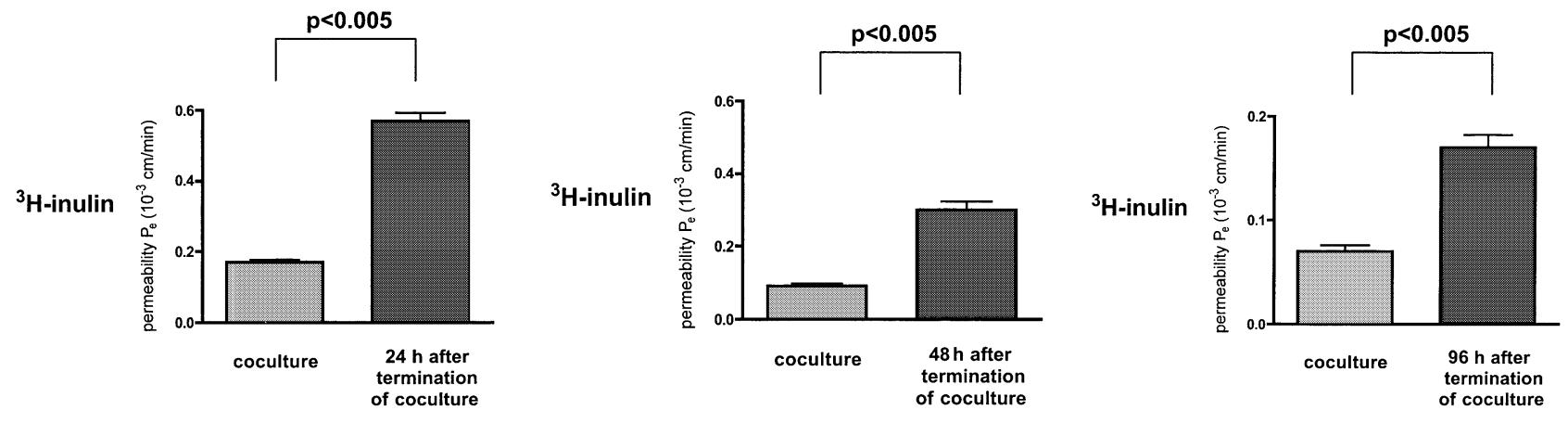

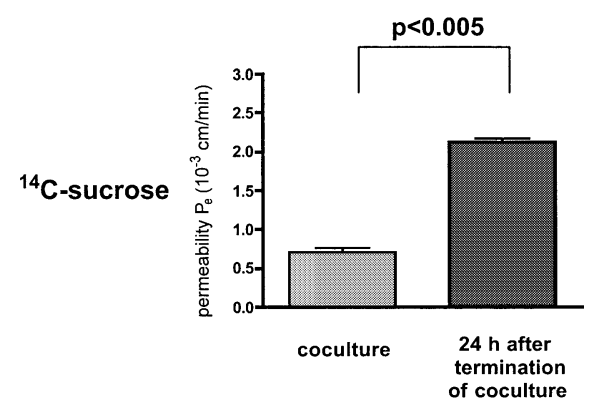

Fig. 2 Modulation of the in vitro BBB. Effect of the co-culture on the maintenance of a permeability barrier across a BBCE cell monolayer. After 10 days of co-culture with astrocytes, BBCE cells were removed from the co-culture and grown in the absence of astrocytes for 1, 2 or 4 consecutive days. Permeability changes in
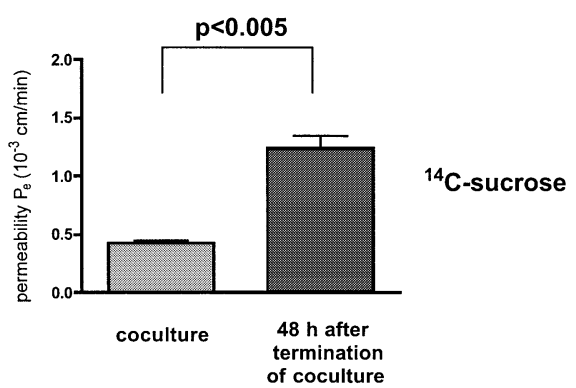

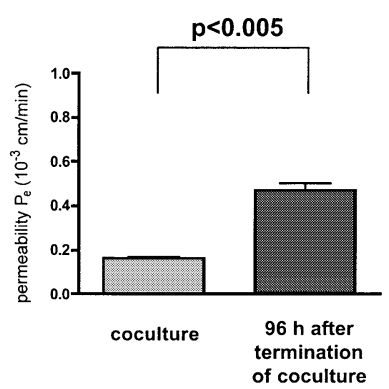

comparison to the co-culture control were measured using ${ }^{14} \mathrm{C}$ sucrose and ${ }^{3} \mathrm{H}$-inulin as tracers. One representative experiment of $n=5$ for two different BBCE clones, namely Lau-1 (24 h) and pJ (48 and $96 \mathrm{~h}$ ), is shown for each timepoint. Bars represent means of triplicates $\pm \mathrm{SE}$

Fig. 3 Actin cytoskeleton of BBCE cells. Actin cytoskeleton of $\mathrm{BBCE}$ cells of the clone $\mathrm{pJ}$ in co-culture with astrocytes and after termination of the co-culture is shown. Immunofluorescence of the actin cytoskeleton with FITC-phalloidin shows a cortical ring in $\mathrm{BBCE}$ cells for both culture conditions. $\times 250$

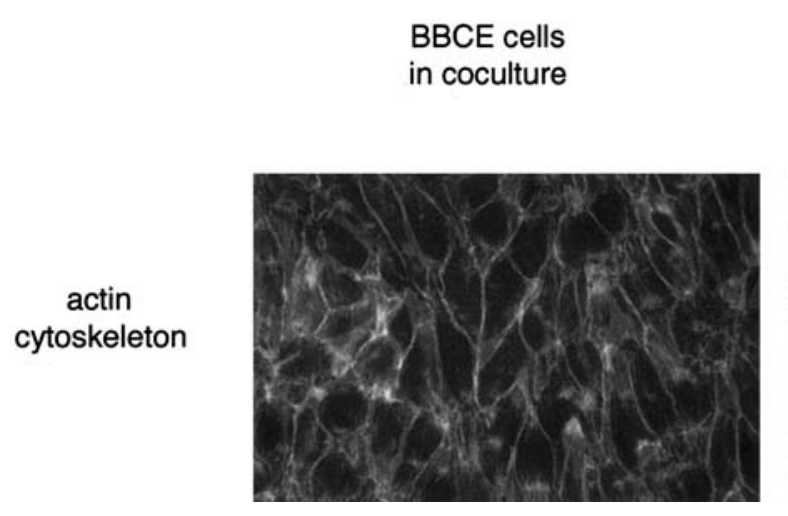

\begin{abstract}
BBCE cells $24 \mathrm{~h}$ after termination of coculture
\end{abstract}

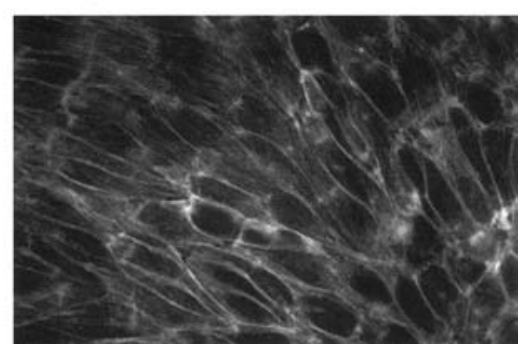

characteristics of BBCE cell monolayers, the paracellular permeabilities for the radioactive tracers ${ }^{3} \mathrm{H}$-inulin and ${ }^{14} \mathrm{C}$-sucrose were always measured within the very same experimental setup. In BBCE cell monolayers grown in co-culture with astrocytes $\left(\mathrm{P}_{\mathrm{e}}\right.$ (inulin) $=$ $0.17 \times 10^{-3} \pm 0.02 \times 10^{-3} \mathrm{~cm} / \mathrm{min}$ and $\mathrm{P}_{\mathrm{e}}$ (sucrose $)=0.51 \times$ $\left.10^{-3} \pm 0.04 \times 10^{-3} \mathrm{~cm} / \mathrm{min}\right)$ the electron-dense reaction product of WGA-HRP was detected at the apical plasma membrane of BBCEs and within rare vesicles along the apical surface (Fig. 4a, b). Furthermore the WGA-HRP reaction product could be seen in interendothelial clefts up to areas where plasma membranes of two adjacent endothelial cells formed a TJ (Fig. 4a, b). In a total of 32 analyzed BBCE cells WGA-HRP reaction product could never be detected below the tight junctional area or at the basolateral surface of the BBCE cells or within the underlying collagen gel. Removal of astrocytes leading to increased permeabilities of BBCE cell monolayers for ${ }^{3} \mathrm{H}-$ inulin and ${ }^{14} \mathrm{C}$-sucrose $\left(\mathrm{P}_{\mathrm{e}}\right.$ (inulin) $=0.51 \times 10^{-3} \pm 0.06 \times$ $10^{-3} \mathrm{~cm} / \mathrm{min}$ and $\mathrm{P}_{\mathrm{e}}($ sucrose $)=1.37 \times 10^{-3} \pm 0.11 \times 10^{-3} \mathrm{~cm} /$ $\min ; p<0.005)$ within $48 \mathrm{~h}$ was associated with an increased diffusion of WGA-HRP through the cell-to-cell contacts: WGA-HRP reaction product was detected without any exception throughout the entire intercellular cleft, additionally along the basolateral membrane in a total of $38 \mathrm{BBCE}$ cells analyzed and within the collagen gel (Fig. 4c, d). Quantification of the number of pinocytotic vesicles in BBCE cells in coculture revealed 48 apical, 36 basolateral and 2 basal vesicles in a total of 32 cells, which averages 2.7 vesicles per cell. Upon 


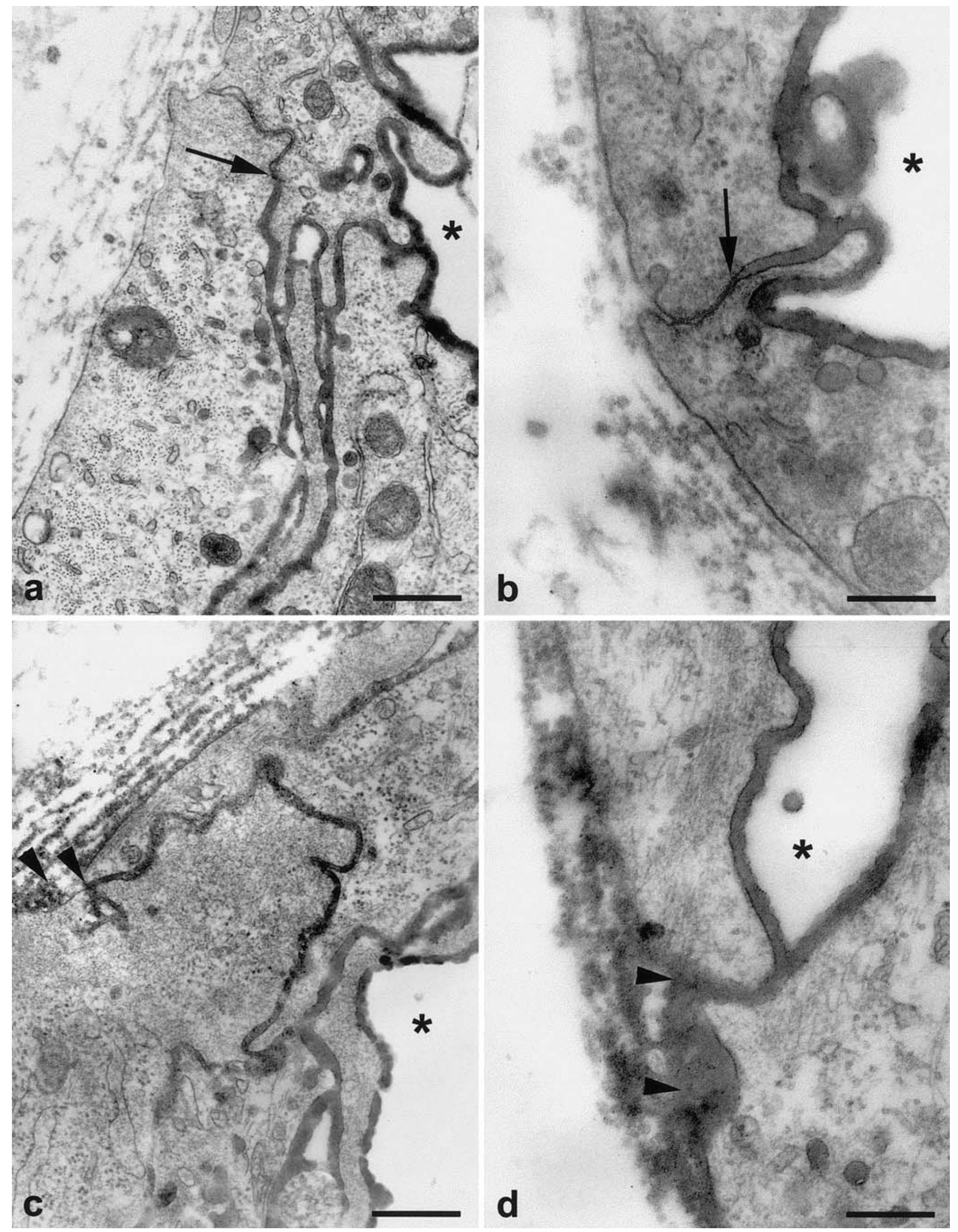


Fig. 5 Subcellular distribution of claudins in BBCE cells. Immunofluorescence staining of BBCE cells in co-culture with astrocytes (left panel) and $24 \mathrm{~h}$ after termination of the co-culture (right panel) are shown. No difference in the subcellular distribution of claudin-3 or claudin-5 can be observed. Staining for claudin-1 did not reveal a junctional staining (not shown). $\times 250$

\section{BBCEs in co-culture}

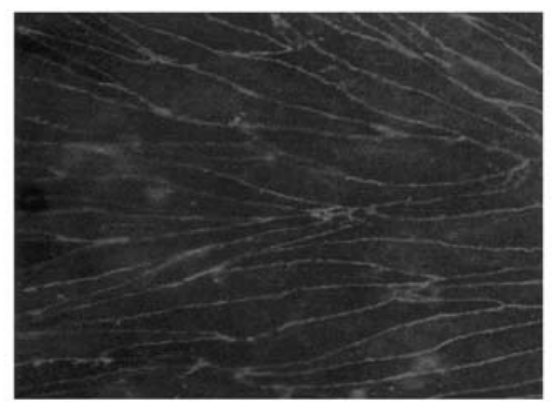

Claudin-3

Claudin-5

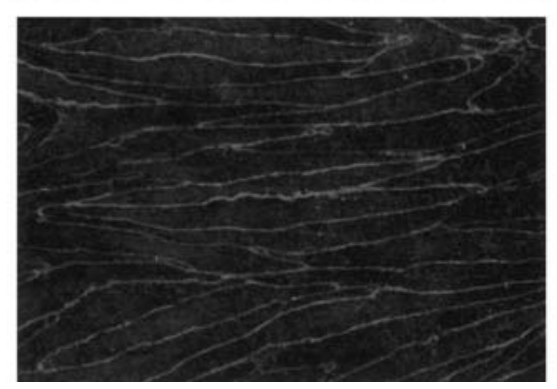

BBCEs 24 hours after termination of coculture
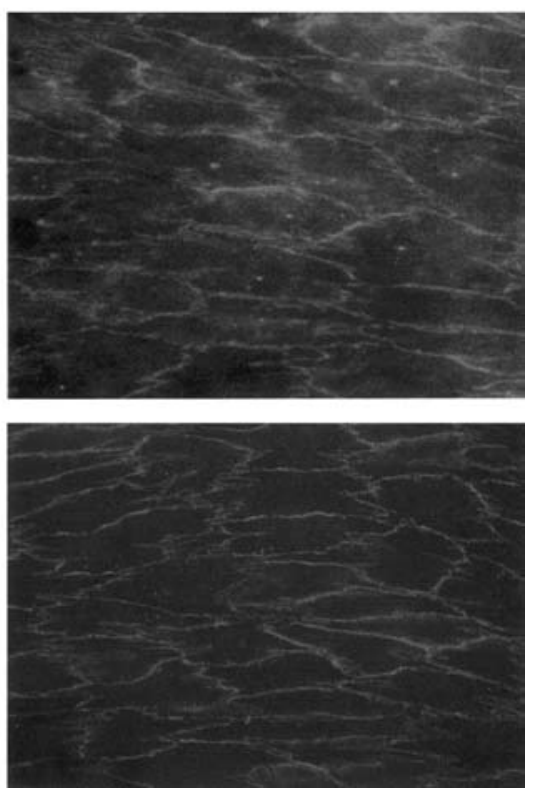

removal of astrocytes vesicle counts revealed 42 on the apical, 58 on the basolateral and 15 on the basal site of 38 counted BBCEs, which averages 3 vesicles per cell. Therefore the number of intracellular vesicles staining positive for the WGA-HRP reaction product was not increased compared to the number of pinocytotic vesicles in BBCE cells in co-culture with astrocytes. Thus, increased permeabilities for ${ }^{3} \mathrm{H}$-inulin and ${ }^{14} \mathrm{C}$-sucrose across BBCE cell monolayers upon removal of astrocytes was accompanied by an opening of BBCE cell TJs to WGA-HRP.

Fig. 4a-d Ultrastructural study of the passage of WGA-HRP across BBCE cell monolayers. Electron micrographs of $\mathrm{BBCE}$ cells (clone $\mathrm{pJ}$ ) cultured on collagen-coated filter inserts are shown. After 12 days of co-culture with astrocytes, co-culture was either continued (a, b) or discontinued (c, d) for two consecutive days. Diffusion of WGA-HRP across the BBCE cell monolayer was investigated for $10 \mathrm{~min}$ after adding WGA-HRP to the upper compartment of the two-chamber system. a, b In the intercellular cleft, the reaction product penetrates from the "luminal" compartment (asterisks) to the TJ which occludes the cleft (arrows). The intercellular cleft from this point to the basal membrane is free of the electron-dense reaction product. c, d After termination of the co-culture there is no occlusion of the intercellular cleft between the BBCE cells, and the tracer is allowed to penetrate from the "lumenal" compartment (asterisks) through the entire intercellular cleft and is deposited in the underlying collagen matrix (arrowheads). Bars $0.5 \mu \mathrm{m}(\mathbf{a}, \mathbf{c}), 0.25 \mu \mathrm{m}(\mathbf{b}, \mathbf{d})$
Subcellular localization of BBB endothelial junction-associated molecules

After demonstrating the opening of the tight junctional barrier at the ultrastructural level, we were interested in whether the modulation of endothelial TJs between BBCE cells upon removal of astrocytes was due to the loss of TJassociated molecules from the cell borders. Therefore, permeability measurements were performed in parallel to immunofluorescence studies for the TJ-associated molecules claudin-1, claudin-3, claudin-5, occludin, ZO-1 and ZO-2 at $24 \mathrm{~h}, 48 \mathrm{~h}$ or $96 \mathrm{~h}$ after termination of the coculture. We also included an analysis of the adherens junction-associated proteins $\beta$-catenin and $120^{\text {cas }}$ as in cerebral endothelial cells non-occluding adherens junctions were found in close proximity to TJs (Schulze and Firth 1993) and were suggested to enhance the occlusive function of TJs.

Immunostaining for claudin-3 and claudin-5 of BBCE cell monolayers derived from co-cultures with astrocytes $\left(\mathrm{P}_{\mathrm{e}}(\right.$ sucrose $\left.)=0.47 \times 10^{-3} \pm 0.07 \times 10^{-3} \mathrm{~cm} / \mathrm{min}\right)$ showed a strict localization for both claudins at the endothelial cell borders (Fig. 5), whereas no junctional staining of BBCEs could be observed with the anti-claudin-1 antibody (data not shown). Immunostaining of BBCE cell monolayers $24 \mathrm{~h}$ after removal of astrocytes revealed no significant changes in the subcellular distribution of claudin-3 and claudin-5 although the paracellular permeability was already dramatically increased $\left(\mathrm{P}_{\mathrm{e}}(\right.$ sucrose $)=$ $\left.0.9 \times 10^{-3} \pm 0.007 \times 10^{-3} \mathrm{~cm} / \mathrm{min}, p<0.05\right)$. Lack of significant changes in the localization of claudin-3 and claudin5 in endothelial cell junctions was confirmed in an effort to quantify junctional stainings of BBCE monolayers. The 
Fig. 6 Subcellular distribution of junction-associated molecules in BBCE cells. Immunofluorescence stainings of BBCE cells in co-culture with astrocytes (left panel) and 4 days after termination of the co-culture (right panel) are shown. No difference in the subcellular distribution of occludin, ZO-1, $\mathrm{ZO}-2, \beta$-catenin and $\mathrm{p} 120^{\text {cat }} \mathrm{can}$ be observed. $\times 300$

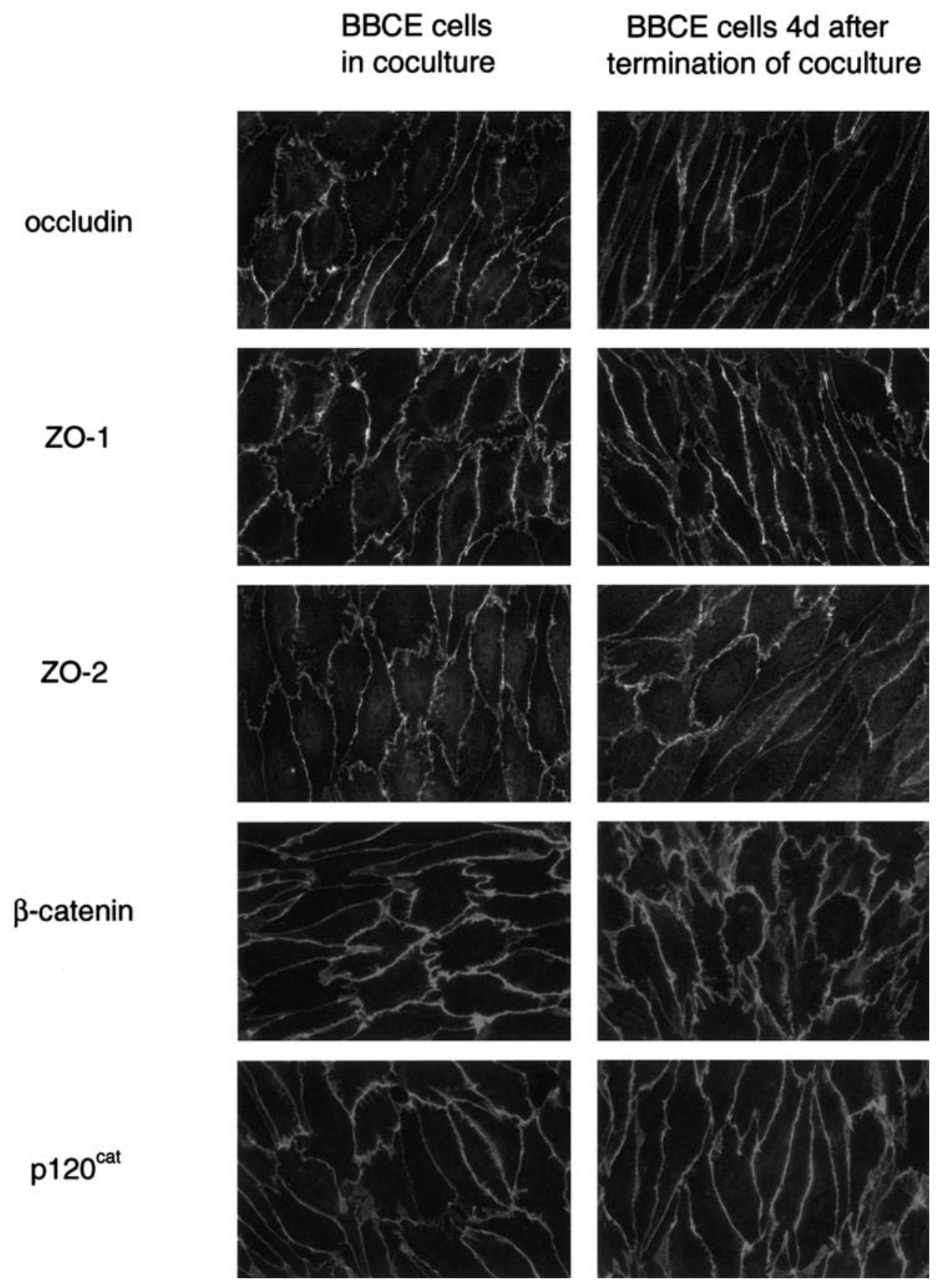

mean number of high intensity pixels for claudin-3 staining was calculated to be $2,659 \pm 1,075$ vs $2,305 \pm 1,093$ $(p>0.5)$ and for claudin-5 2,155 \pm 930 vs $2,712 \pm 1,143$ $(p>0.45)$ when comparing BBCEs in coculture with astrocytes vs BBCEs $24 \mathrm{~h}$ after termination of coculture, respectively.

Furthermore occludin, ZO-1, ZO-2, $\beta$-catenin and p120 cas were exclusively detected at the cell-to-cell contacts of BBCE cells grown in co-cultures with astrocytes $\left(\mathrm{P}_{\mathrm{e}}\right.$ (inulin) $=0.04 \times 10^{-3} \pm 0.01 \times 10^{-3} \mathrm{~cm} / \mathrm{min}$ and $\mathrm{P}_{\mathrm{e}}($ sucrose $\left.)=0.09 \times 10^{-3} \pm 0.01 \times 10^{-3} \mathrm{~cm} / \mathrm{min}\right)($ Fig. 6). Immunofluorescence studies of BBCE cells $96 \mathrm{~h}$ after termination of the co-culture $\left(\mathrm{P}_{\mathrm{e}}(\right.$ inulin $)=0.10 \times 10^{-3} \pm$ $0.02 \times 10^{-3} \mathrm{~cm} / \mathrm{min}, p<0.01$, and $\mathrm{P}_{\mathrm{e}}($ sucrose $)=0.26 \times 10^{-3} \pm$ $\left.0.03 \times 10^{-3} \mathrm{~cm} / \mathrm{min}, p<0.001\right)$ showed also no visible change in the subcellular distribution of these molecules (Fig. 6 and not shown). Lack of significant changes in the localization of junctional proteins was confirmed by an effort to quantify junctional staining of $\beta$-catenin in BBCE monolayers. The mean number of high intensity pixels for $\beta$-catenin staining was calculated to be $14,959 \pm 2,564$ vs $17,584 \pm 5,116(p=0.4)$ when comparing BBCEs in coculture with astrocytes versus BBCEs 4 days after termination of coculture, respectively. Thus, in vitro an increase in paracellular permeability across BBCE monolayers is not readily accompanied by the visible loss of a tight junction protein from the cell-to-cell contact. 


\section{Discussion}

The fully differentiated BBB consists of a complex cellular system of highly specialized endothelial cells, a high number of pericytes embedded in the basal membrane, perivascular macrophages and astrocytic endfeet. While the endothelial cells form the barrier proper, the interaction with adjacent cells seems to be a prerequisite for barrier function. The complex tight junctions (TJs) between endothelial cells are primarily responsible for the barrier function and unlike simple TJs provide a high electrical resistance (about 2,000 $\Omega \times \mathrm{cm}^{2}$ ) (Crone and Olesen 1982). The molecular changes involved in dysfunction of BBB endothelial TJs have not yet been characterized. Several studies have suggested that TJ integrity is dependent on the structural organization of the TJ-associated proteins and their interaction with the actin cytoskeleton. Thus, breakdown of the BBB as observed in CNS disorders might be associated with an alteration of the molecular composition of BBB TJs. This notion is supported by observations made by us and others. We have observed a selective loss of claudin-3, but not claudin-5, ZO-1 or occludin, from BBB TJs in altered blood vessels in human glioblastoma multiforme and in experimental autoimmune encephalomyelitis (Wolburg et al. 2003), whereas leaky blood vessels of the CNS in dystrophic mdx mice have been shown to demonstrate a loss of junctional localization of $\mathrm{ZO}-1$ and claudin-1 (Nico et al. 2003).

In order to investigate molecular changes at cerebral TJs associated with the development of BBB leakiness, we established an in vitro BBB model which would allow us to correlate expression and subcellular localization of TJ molecules in cerebral endothelial cells with their barrier characteristics. Several in vitro BBB models have been established which utilize the co-culture of cerebral porcine or bovine brain endothelial cells with primary astrocytes mimicking the in vivo BBB microenvironment (Meresse et al. 1989; Rubin et al. 1991; Wolburg et al. 1994). In this study we decided to employ an in vitro BBB model, in which bovine brain endothelial (BBCE) cells are cocultured with primary rat astrocytes and which has proven to maintain many $\mathrm{BBB}$ characteristics in addition to a permeability barrier (reviewed in Cecchelli et al. 1999). In agreement with numerous previous studies (reviewed in Wolburg and Lippoldt 2002), BBCE cells grown in monoculture in vitro did not build a permeability barrier to tracers, whereas co-culture of BBCE cells with primary astrocytes for 10 days induced a tight permeability barrier. We furthermore demonstrate here that removal of astrocytes from the co-culture resulted in an increased permeability for tracers across the barrier built by BBCE cells. Therefore in this in vitro system the permeability barrier of the BBCE cells was directly dependent on humoral factors present in the co-culture only. Modulation of the BBB in vitro was due to the opening of the endothelial TJs as demonstrated by an ultrastructural analysis using horseradish peroxidase. An increase in the number of pinocytotic vesicles leading to an increased transendothelial transport of tracers did not occur.
In cerebral endothelial cells tight and adherens junctions have been described to be intercalated (Schulze and Firth 1993). This has been supported by the finding that the TJ protein claudin-5 precisely colocalizes with the adherens junction protein VE-cadherin (Morita et al. 1999b). In addition, expression and localization of $\beta$ catenin and $\mathrm{p} 120^{\text {cas }}$ have been described to be crucial for the functional state of adherens junctions in endothelial cells (Dejana 1996). Therefore we investigated the subcellular localization of the adherens junction molecules $\beta$-catenin and $\mathrm{p} 120^{\text {cas }}$ in BBCE cells in correlation to the permeability barrier formed in vitro. We did not observe any changes in the subcellular distribution of $\beta$ catenin and $\mathrm{p} 120^{\mathrm{cas}}$ in correlation to permeability changes in vitro, suggesting that there is no major alteration in the molecular architecture of adherens junctions.

The observed maintenance of the cortical actin belt in BBCE cells after removal of astrocytes further supports the view that opening of the endothelial junctions is not mediated by a general loss of junction-associated molecules from the cell-to-cell contacts of BBCE cells. This notion is also underlined by our observations that the subcellular distribution of the TJ-associated proteins ZO-1, ZO-2, occludin, claudin-3 and claudin-5 did not change during permeability changes in vitro. A lack of sensitivity of the immunostaining to visualize a change in the localization of TJ-associated proteins seems unlikely since we have previously demonstrated that a 30-min treatment of BBCEs with mannitol, a mannose-derived sugar alcohol commonly used to increase paracellular permeability across the BBB (Rapoport and Robinson 1986), resulted in an increase in the paracellular permeability across BBCE cell monolayers and under these conditions occludin staining showed disruptions and large gaps between the BBCE cells (Brillaut et al. 2002). In the experiments performed in the present study, no gaps were observed in between BBCE cells and permeability increase values were less prominent than in the mannitol treatment.

Using immunostaining techniques, we did not find any visible loss of TJ molecules from endothelial junctions, correlating with the early opening of the TJs to paracellular tracers in vitro. Therefore our data suggest that paracellular permeability is not simply controlled by the presence or absence of TJ-associated proteins within the junctions. This is at least true during embryonic development, where claudin-5 is detected in cerebral endothelial TJs at early timepoints during brain angiogenesis well before the formation and maturation of the BBB, demonstrating that maturation of the permeability barrier is not attributable to the developmental changes in the expression of claudin-5. In contrast, during pathological conditions within the CNS such as glioblastoma multiforme or CNS inflammation, the selective loss of claudin3 , but not of claudin-5, ZO-1 or occludin, from altered cerebral vessels was observed by us previously (Wolburg et al. 2003), suggesting that loss of TJ proteins from cellto-cell contacts can be a late event in BBB breakdown.

Taken together, our in vitro data demonstrate that surprisingly $\mathrm{TJ}$-associated proteins retain their localization at the endothelial $\mathrm{TJ}$ in the face of an increase in 
paracellular permeability. This seems to be in apparent contrast to in vivo observations, where a complete loss of TJ-associated proteins from the BBB TJs was observed in long established pathological conditions accompanied by BBB breakdown. We thus conclude that loss of localization of TJ-associated proteins from the BBB TJs is a relatively late event, which is not yet observed in the comparatively short term in vitro experiments performed here. Consequently, early and more subtle changes at the TJ distinct from the complete loss of TJ-associated proteins, i.e., their phosphorylation, cause the increase in paracellular permeability observed in the present study. The molecular nature of these changes will have to be investigated in more detail.

Acknowledgements We thank Urban Deutsch, Friedemann Kiefer and Dietmar Vestweber for helpful discussions with the manuscript.

\section{References}

Ando-Akatsuka Y, Saitou M, Hirase T, Kishi M, Sakakibara A, Itoh M, Yonemura S, Furuse M, Tsukita S (1996) Interspecies diversity of the occludin sequence: cDNA cloning of human, mouse, dog, and rat-kangaroo homologues. J Cell Biol 133:4347

Booher J, Sensenbrenner M (1972) Growth and cultivation of dissociated neurons and glial cells from embryonic chick, rat and human brain in flask cultures. Neurobiology 2:97-105

Brillaut J, Berezowsky V, Cecchelli R, Dehouck MP (2002) Intercommunications between brain capillary endothelial cells and glial cells increase the transcellular permeability of the blood-brain barrier during ischemia. J Neurochem 83:807-817

Cecchelli R, Dehouck B, Descamps L, Fenart L, Buee-Scherrer V, Duhem C, Lundquist S, Rentfel M, Torpier G, Dehouck MP (1999) In vitro model for evaluating drug transport across the blood-brain barrier. Adv Drug Deliv Rev 36:165-178

Crone C, Olesen SP (1982) Electrical resistance of brain microvascular endothelium. Brain Res 241:49-55

Dehouck MP, Meresse S, Delorme P, Fruchart JC, Cecchelli R (1990) An easier, reproducible, and mass-production method to study the blood-brain barrier in vitro. J Neurochem 54:17981801

Dejana E (1996) Endothelial adherens junctions: implications in the control of vascular permeability and angiogenesis. J Clin Invest 98:1949-1953

Descamps L, Cecchelli R, Torpier G (1997) Effects of tumor necrosis factor on receptor-mediated endocytosis and barrier functions of bovine brain capillary endothelial cell monolayers. J Neuroimmunol 74:173-184

Engelhardt B, Risau W (1995) The development of the blood-brain barrier. In: Greenwood J, Begley D, Segal M, (eds) New concepts of a blood-brain barrier. Plenum Press, London

Farqhuar MG, Palade GE (1963) Junctional complexes in various epithelia. J Cell Biol 17:375-412

Furuse M, Hirase T, Itoh M, Nagafuchi A, Yonemura S, Tsukita S (1993) Occludin - a novel integral membrane-protein localizing at tight junctions. J Cell Biol 123:1777-1788

Furuse M, Sasaki H, Fujimoto K, Tsukita S (1998) A single gene product, claudin- 1 or -2 , reconstitutes tight junction strands and recruits occludin in fibroblasts. J Cell Biol 143:391-401

Furuse M, Sasaki H, Tsukita S (1999) Manner of interaction of heterogenous claudin species within and between tight junction strands. J Cell Biol 147:891-903

Goldstein GW (1988) Endothelial cell-astrocyte interactions. A cellular model of the blood-brain barrier. Ann N Y Acad Sci 529:31-39
Gumbiner B, Simons K (1986) A functional assay for proteins involved in establishing an epithelial occluding barrier: identification of an uvomorulin-like polypeptide. J Cell Biol 102:457-468

Kniesel U, Wolburg H (2000) Tight junctions of the blood-brain barrier. Cell Mol Neurobiol 20:57-76

Liebner S, Kniesel U, Kalbacher H, Wolburg H (2000a) Correlation of tight junction morphology with the expression of tight junction proteins in blood-brain barrier endothelial cells. Eur J Cell Biol 79:707-717

Liebner S, Fischmann A, Rascher G, Duffner F, Grote E-H, Kalbacher H (2000b) Claudin-1 and claudin-5 expression and tight junction morphology are altered in blood vessels of human glioblastoma multiforme. Acta Neuropathol 100:323-331

Meresse S, Dehouck MP, Delorme P, Bensaid M, Tauber JP, Delbart C, Fruchart JC, Cecchelli R (1989) Bovine brain endothelial cells express tight junctions and monoamine oxidase activity in long-term culture. J Neurochem 53:13631371

Mitic LL, Van Itallie CM, Anderson JM (2000) Molecular physiology and pathophysiology of tight junctions. I. Tight junction structure and function: lessons from mutant animals and proteins. Am J Physiol 279:G250-G254

Morita K, Furuse M, Fujimoto K, Tsukita S (1999a) Claudin multigene family encoding four-transmembrane domain protein components of tight junction strands. Proc Natl Acad Sci U S A 96:511-516

Morita K, Sasaki H, Furuse M, Tsukita S (1999b) Endothelial claudin: claudin-5/TMVCF constitutes tight junction strands in endothelial cells. J Cell Biol 147:185-194

Nico B, Frigeri A, Nicchia GP, Corsi P, Ribatti D, Quondamatteo F, Herken R, Girolamo F, Marzullo A, Svelto M, Roncali L (2003) Severe alterations of endothelial and glial cells in the bloodbrain barrier of dystrophic mdx mice. Glia 42:235-51

Phelps CH (1972) The development of glio-vascular relationships in the rat spinal cord. An electron microscopic study. Z Zellforsch Mikrosk Anat 128:555-563

Rapoport SI, Robinson PJ (1986) Tight junctional modifications as the basis of osmotic opening of the blood-brain barrier. Ann N Y Acad Sci 481:250-267

Rubin LL, Hall DE, Porter S, Barbu K, Cannon C, Horner HC, Janatpour M, Liaw CW, Manning K, Morales J, Tanner LI, Tomaselli KJ (1991) A cell-culture model of the blood-brain barrier. J Cell Biol 115:1725-1735

Saitou M, Furuse M, Sasaki H, Schulzke J-D, Fromm M, Takano H, Noda T, Tsukita S (2000) Complex phenotype of mice lacking occludin, a component of tight junction strands. Mol Biol Cell 22:4131-4142

Schulze C, Firth JA (1993) Immunohistochemical localization of adherens junction components in blood-brain-barrier microvessels of the rat. J Cell Sci 104:773-782

Siflinger-Birnboim A, Del Vecchio PJ, Ciooper JA, Blumenstock FA, Shepard JN, Mailik AB (1987) Molecular sieving characteristics of the cultured endothelial monolayer. J Cell Physiol 132:111-117

Tsukita S, Furuse M, Itoh M (1999) Structural and signalling molecules come together at tight junctions. Curr Opin Cell Biol $11: 628-633$

Wolburg H, Lippoldt A (2002) Tight junctions of the blood-brain barrier: Development, composition and regulation. Vasc Pharmacol 38:323-337

Wolburg H, Neuhaus J, Kniesel U, Krauss B, Schmid EM, Ocalan M, Farrell C, Risau W (1994) Modulation of tight junction structure in blood-brain-barrier endothelial-cells-effects of tissue-culture, 2nd messengers and co-cultured astrocytes. J Cell Sci 107:1347-1357

Wolburg H, Wolburg-Buchholz K, Kraus J, Rascher-Eggstein G, Liebner S, Hamm S, Duffner F, Grote E-H, Risau W, Engelhardt B (2003) Localization of claudin-3 in tight junctions of the blood-brain barrier is selectively lost during experimental autoimmune encephalomyelitis and human glioblastoma multiforme. Acta Neuropathol 105:586-592 